\title{
Longitudinal transport measurements in an energy recovery accelerator with triple bend achromat arcs
}

\author{
F. Jackson, ${ }^{*}$ D. Angal-Kalinin, Y. M. Saveliev, and P. H. Williams \\ ASTeC, STFC Daresbury Laboratory and Cockcroft Institute, Warrington WA4 4AD, United Kingdom
}

A. Wolski

Department of Physics, University of Liverpool, Liverpool L69 7ZE, and Cockcroft Institute, Warrington WA4 4AD, United Kingdom

(Received 8 July 2016; published 23 December 2016)

\begin{abstract}
Longitudinal properties of electron bunches (energy spread and bunch length) and their manipulation are of importance in free electron lasers (FELs), where magnetic bunch length compression is a common feature of beam transport. Recirculating accelerators and energy recovery linac accelerators (ERLs) have been used as FEL drivers for several decades and control of longitudinal beam transport is particularly important in their magnet lattices. We report on measurements of longitudinal transport properties in an ERL-FEL, the ALICE (Accelerators and Lasers in Combined Experiments) accelerator at Daresbury Laboratory. ALICE is an energy recovery research accelerator that drives an infrared free electron laser. By measuring the time of arrival of electron bunches, the canonical longitudinal transport quantities were measured in the beam transport and bunch compression sections of the lattice. ALICE includes a fourdipole bunch compression chicane providing fixed longitudinal transport, and triple bend achromat arcs including sextupole magnets where the first and second order longitudinal transport can be adjusted. The longitudinal transport properties in these lattice sections were measured and compared with the theoretical model of the lattice. A reasonable level of agreement has been found. The effect of sextupoles in second order, as well as first order, longitudinal correction is considered, with the measurements indicating the level of alignment of the beam to the center of the sextupole.
\end{abstract}

DOI: 10.1103/PhysRevAccelBeams.19.120701

\section{INTRODUCTION}

The ALICE facility is an energy recovery accelerator which drives an infrared free electron laser (IR-FEL). It consists of a dc electron gun, a superconducting booster module, and an energy recovery loop containing a superconducting linac, a bunch compressor and an undulator. The dc photoinjector gun produces sub-MeV electrons which are bunched with a normal-conducting rf cavity, boosted to a few $\mathrm{MeV}$ by the first superconducting module, and injected into the linac where they are accelerated up to around $30 \mathrm{MeV}$. The machine layout is shown in Fig. 1. Bunch compression is achieved by running the main linac off-crest to give a momentum chirp to the bunch, followed by transport through a nominally isochronous arc and a four-dipole chicane. The beam is then passed through the IR-FEL undulator and a nonisochronous decompressing arc to return the beam to the linac for energy recovery; the first arc is mechanically translatable to enable net path length variation. Bunches with charge up to $100 \mathrm{pC}$ are

\footnotetext{
frank.jackson@stfc.ac.uk

Published by the American Physical Society under the terms of the Creative Commons Attribution 3.0 License. Further distribution of this work must maintain attribution to the author $(s)$ and the published article's title, journal citation, and DOI.
}

used. Bunch repetition rates (within a bunch train) can be varied up to $81.25 \mathrm{MHz}$, with train lengths up to $100 \mu \mathrm{s}$ and train repetition rates up to $10 \mathrm{~Hz}$. The fundamental $\mathrm{rf}$ frequency is $1.3 \mathrm{GHz}$.

Longitudinal dynamics (bunch length and energy spread and their evolution) are of importance in ALICE since short bunches of $\approx 1 \mathrm{ps}$ with energy spread $\approx 0.7 \%$ are required for the operation of the IR-FEL [1]. Short bunches are also important for the production of coherent terahertz radiation in the compression chicane, which is another application of the ALICE facility [2]. The bunch compression is further enhanced by means of higher order magnetic corrections, made possible by sextupoles in the first arc which correct the curvature of the momentum chirp.

The aim of the studies presented here was to investigate experimentally the longitudinal beam dynamics and bunch compression, via measurements of the bunch time of flight, which can give access to the properties of the longitudinal transfer map. In the following sections we set out the principles of the theory and measurement method, and compare results with expectations. The results yield a characterization of the longitudinal properties of the triple bend achromat (TBA) arcs and compression chicane used in ALICE, and the issues associated with sextupole correction within these arcs.

Although other ERL-driven FELs have been developed and operated, published measurements of their longitudinal 


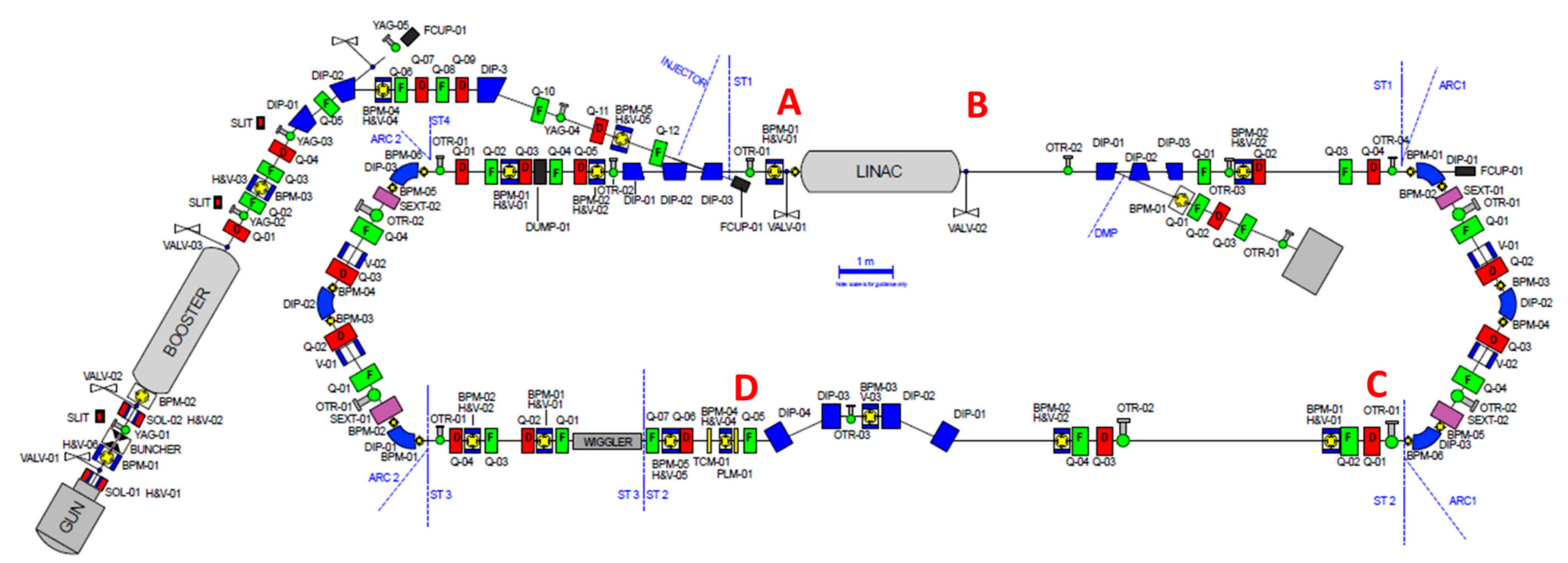

FIG. 1. Layout of the ALICE energy recovery linac accelerator (ERL) showing locations A (main linac entrance), B (main linac exit), $\mathrm{C}$ (first arc exit) and D (compression chicane exit).

transport properties are rare. Similar studies to those presented here have been performed at the ERL IR-FEL at Jefferson Laboratory [3], where the transport arcs used are not TBA but the "Bates bend" design. At the S-DALINAC recirculating accelerator at Darmstadt (which uses similar arcs to the TBA of ALICE), longitudinal properties have been measured with the aim of reducing the energy spread of the extracted beam. The measurements at the S-DALINAC [4] share some similarities to those presented here. However, we are not aware of any experimental investigation of both first and second order transport maps of the TBA arc system including sextupoles.

There are other comparable ERL machines of interest for which longitudinal dynamics are important. They include the ERL IR-FEL developed by the Japan Atomic Energy Agency (JAEA) [5] based on TBA arcs. More recently another compact ERL has been commissioned in Japan at KEK, also using TBA arcs including sextupoles at which longitudinal dynamics and bunch compression have been subjects of recent study [6,7]. The Novosibirsk ERL-FEL [8], the only multiturn ERL, shares some basic similarities with ALICE although it operates in a somewhat different beam and FEL parameter regime. Finally a prototype high current ERL is currently being constructed at HZB, Germany (Helmholtz-Zentrum Berlin für Materialien und Energie), which will have a short pulse mode requiring bunch compression relying entirely on longitudinal properties of TBA arcs $[9,10]$.

Elsewhere, the results reported in this paper may have relevance for machines that do not use the ERL principle but contain loops or arcs and for which longitudinal properties are important, for example the IR-FEL at Kyoto University [11].

\section{MAGNETIC BUNCH COMPRESSION}

We briefly describe the theory of magnetic bunch compression in the ideal case, which relies on a correlation between particle momentum and longitudinal coordinate in the bunch (the head of the bunch is at a different momentum to the tail). If a bunch with an appropriate correlation passes through a section of beam line in which the distance traveled by a particle depends on its momentum, the head-tail separation can be decreased.

The longitudinal dynamics can be described in terms of the momentum deviation $\delta=\left(P-P_{0}\right) / P_{0}$ (where $P$ is the particle momentum and $P_{0}$ is the reference momentum), and longitudinal coordinate $z$ (which describes the position of a particle in a bunch relative to a reference particle traveling along the beam line with the reference momentum). The transfer map for the momentum deviation from the linac entrance (point $\mathrm{A}$ in Fig. 1) to the linac exit (point B) can be written (to second order)

$$
\delta_{B}=R_{66} \delta_{A}+R_{65} z_{A}+T_{655} z_{A}^{2}
$$

where $R_{66}$ represents the effect of the adiabatic damping in the linac, $R_{65}$ represents the momentum chirp, and $T_{655}$ represents the effect of the rf curvature. It is assumed that the longitudinal coordinate $z$ of the particle does not change in the linac (relativistic approximation).

The transfer map for the longitudinal coordinate from the linac entrance (point A) to the compression chicane exit (point D) is

$$
z_{D}=z_{A}+R_{56} \delta_{B}+T_{566} \delta_{B}^{2},
$$

where $R_{56}$ and $T_{566}$ represent the linear and second order dependence of path length on momentum deviation from the arc and the chicane. The longitudinal transfer matrix elements $R_{56}$ and $T_{566}$ are often referred to as the (first and second order, respectively) momentum compaction or longitudinal dispersion.

From (1) and (2) it follows that the final mean square bunch length is given by 


$$
\begin{aligned}
\left\langle z_{D}^{2}\right\rangle= & \left(1+R_{56} R_{65}\right)^{2}\left\langle z_{A}^{2}\right\rangle \\
& +2 R_{56} R_{66}\left(1+R_{56} R_{65}\right)\left\langle z_{A} \delta_{A}\right\rangle \\
& +R_{56}^{2} R_{66}^{2}\left\langle\delta_{A}^{2}\right\rangle \\
& +O(3),
\end{aligned}
$$

where $\left\langle z_{A}^{2}\right\rangle$ is the initial mean square bunch length, $\left\langle\delta_{A}^{2}\right\rangle$ is the initial mean square momentum spread, $\left\langle z_{A} \delta_{A}\right\rangle$ is the initial momentum chirp, and $O(3)$ represents terms of third order and above in the variables $z_{A}$ and $\delta_{A}$.

In the case where the bunch injected into the linac has no chirp and the initial momentum spread is much smaller than the initial bunch length, so that

$$
\sqrt{\left\langle\delta_{A}^{2}\right\rangle} \ll \frac{1+R_{56} R_{65}}{R_{56} R_{66}} \sqrt{\left\langle z_{A}^{2}\right\rangle},
$$

then the final bunch length (3) can be minimized (to second order) by enforcing the condition

$$
R_{65}=-1 / R_{56}
$$

The quantity $R_{65}$ describing the chirping transformation by the $\mathrm{rf}$ is given in terms of the acceleration parameters:

$$
R_{65}=-\frac{e V_{r f} \sin \left(\phi_{r f}\right) k_{r f}}{e V_{r f} \cos \left(\phi_{r f}\right)+E_{A}},
$$

where $E_{A}$ is the reference energy before acceleration, $V_{r f}$ is the accelerating voltage, $k_{r f}=2 \pi / \lambda_{r f}$ where $\lambda_{r f}$ is the $\mathrm{rf}$ wavelength, and $\phi_{r f}$ is the rf phase. Thus Eqs. (5) and (6) determine the off-crest $\mathrm{rf}$ phase at which maximum compression is achieved. If there is no initial chirp (so that $\left\langle z_{A} \delta_{A}\right\rangle=0$ ) and the conditions (4) and (5) are satisfied, then the final rms bunch length is given by

$$
\sqrt{\left\langle z_{D}^{2}\right\rangle}=R_{56} R_{66} \sqrt{\left\langle\delta_{A}^{2}\right\rangle}+O(3 / 2) .
$$

Higher order terms can lead to significant distortion of the longitudinal phase space at the exit of the chicane and if not properly controlled can result in a bunch that is significantly longer than would be expected by considering only the linear coefficients in the transfer map. The compressed bunch length $\left\langle z_{D}^{2}\right\rangle$ in Eq. (3) can be computed explicitly to third order. Typically, it is found that the dominant higher order term is the term $\left\langle z_{A}^{2} \delta_{A}\right\rangle$; the coefficient of this term can be set to zero by enforcing the condition

$$
T_{566}=-R_{56}^{3} T_{655} .
$$

The values of $R_{56}$ and $T_{655}$ are fixed by the optimization of the linear part of the transfer map. To avoid higher order distortions $T_{566}$ is controlled by including sextupoles at locations in the beam line where the dispersion is nonzero.

\section{ALICE LATTICE LONGITUDINAL TRANSPORT DESIGN AND IMPLEMENTATION}

The ALICE accelerator was originally devised as a prototype fourth generation light source, and its design was influenced by a similar demonstration project, the IR-FEL DEMO at Jefferson Laboratory (JLAB) [12]. The ALICE design is described in detail elsewhere [13-15] and here we just outline the main features.

As described above, the longitudinal transport and bunch compression in ALICE are dominated by a four-dipole magnetic chicane, in similar way to that in DEMO at JLAB. However, the ALICE arcs were chosen to be a triple bend achromat (TBA) design in contrast to the Bates bend of DEMO. Earlier design work on TBA arcs for the CTF3 project at CERN [16] showed that this type of arc could be tuned to provide isochronous transport or to provide nonzero $R_{56}$ [14]. The TBA arc was therefore adopted for ALICE with the first (outward) arc designed to be nominally isochronous and for the second (return) arc designed to cancel the $R_{56}$ of the chicane, thus making the entire energy recovery loop nominally isochronous.

The TBA arcs in ALICE each contain two symmetrically located pairs of quadrupoles (see Fig. 1). The longitudinal transport properties of the arcs are sensitive to the field strength of the outer pair of quadrupoles, allowing them to operate with $R_{56}$ ranging from small positive to large negative values. Note that the $R_{56}$ of the chicane is defined here to be negative: different simulation codes use different conventions, for example MADX [17] defines the $R_{56}$ of such a chicane to be positive, whereas ELEGANT [18] defines it to be negative. The arcs can be made isochronous and achromatic (the so-called "closed" arc condition) for a single unique value of the strengths of the quadrupole pairs. Isochronicity and achromaticity can be achieved nonsimultaneously for other values of the quadrupoles.

The compression chicane (which bends in the horizontal plane) is designed to provide a fixed $R_{56}$ of $-0.28 \mathrm{~m}$. The chicane is composed of four rectangular dipoles angled at half the bend angle (the bend angle is $21.5^{\circ}$ ). For this type of chicane it can be shown that the transfer matrix elements $R_{51}$ and $R_{52}$ are zero (a similar property to a four-dipole chicane with normal entrance and exit of the beam to each dipole [19]). A consequence of this is that the contribution of the chicane $R_{56}$ to the longitudinal transport between the linac and the FEL is simply to add $-0.28 \mathrm{~m}$ to the $R_{56}$ of the upstream lattice, irrespective of whether the arc is closed or not. In other words, all bunch compression flexibility resides in the first arc (and linac chirp) rather than the chicane.

In ALICE the chirp required for compression is provided by the main linac. For typical ALICE parameters 
$\left(k_{r f}=27.2 \mathrm{~m}^{-1}, \quad E_{A} \approx 6.5 \mathrm{MeV}, e V_{r f} \approx 20 \mathrm{MeV}\right)$ and assuming the nominal post-linac $R_{56}$ of $-0.28 \mathrm{~m}$, the phase required for maximum compression, using Eqs. (5) and (6) is approximately $10^{\circ}$. In practice, the FEL performance is often found to be optimized by operating the linac some degrees further off-crest. This is most likely due to an existing reverse chirp on the bunch at the entrance to the linac: there is some evidence for this from simulations and measurements.

At the design phase of $10^{\circ}$, the higher order coefficient describing the linac transformation $T_{655}$ is approximately $270 \mathrm{~m}^{-2}$ and thus the post-linac $T_{566}$ required to cancel this higher order distortion using Eq. (8) is approximately $-6 \mathrm{~m}$. The sextupoles in the first arc, when used in concord at their maximum strength can provide $T_{566}=-20 \mathrm{~m}$ in the longitudinal transport to the FEL. Horizontal misalignment of the sextupoles can lead to a modification of the $R_{56}$ of the arc. The largest components of sextupole field for a horizontally displaced beam are dipolar and quadrupolar, and the quadrupole component may affect the arc $R_{56}$ in a similar fashion to the arc outer quadrupoles.

The operational setup of ALICE is guided by the theory and calculations above for maximum bunch compression. In practice, however, rather than rigorously applying this prescription, the flexibility of the longitudinal transport of the arc allows pragmatic optimization of the FEL radiation intensity by scanning the quadrupole and sextupole strengths to find the optimal working point. The rf phase is also usually tuned (iteratively with the arc settings) to maximize the FEL intensity. To optimize FEL performance, minor adjustments are made to a range of other machine parameters including injector settings, steering and focusing in the undulator, nonundulator steering, and even the beam energy. Beam energy adjustments may affect the longitudinal transport, as will be seen below.

\section{EXTRACTION OF LONGITUDINAL MATRIX ELEMENTS FROM MEASUREMENTS}

The longitudinal transfer matrix elements between any two locations $a$ and $b$ in the lattice may be expressed as $R_{56}=\frac{\partial z_{b}}{\partial \delta_{a}}$, and $T_{566}=\frac{1}{2} \frac{\partial^{2} z_{b}}{\partial \delta_{a}^{2}}$, where the derivatives are evaluated at $\delta=0$; in other words $R_{56}$ and $T_{566}$ are the local gradient and parabolic curvature describing the change in particle longitudinal position as a function of momentum deviation, about the reference momentum $P_{0}$.

Thus for a chosen machine beam momentum set point (typical long term value for ALICE is $P_{0}=26.5 \mathrm{MeV} / c$ ), the values of $R_{56}$ and $T_{566}$ between two arbitrary locations can be found by measuring the change in bunch path length between these locations as functions of the beam momentum, around the set point. This can be achieved by measuring the time of arrival of a bunch (converted to path length simply by multiplying by the speed of light) while varying the beam momentum (via the linac gradient).
In ALICE the measurements of greatest interest for the bunch compression are the values of $R_{56}$ and $T_{566}$ in the compression section of the lattice, i.e. from the linac to the exit of the compression chicane. Also of interest (for characterizing the properties of the TBA lattice) were the values of $R_{56}$ and $T_{566}$ only of the arc.

The path lengths from linac to arc exit and from linac to chicane exit were measured by the time of arrival (TOA) of a bunch (the first bunch in the train) using the signals from beam position monitors (BPMs) at the exit of the first arc and at the chicane exit. Since only path length changes (with respect to changes in beam momentum) are required to obtain $R_{56}$ and $T_{566}$ the absolute time of flight from the linac to these locations is not required.

The TOA is measured by sampling the BPM signal waveforms on a high bandwidth oscilloscope (sampling at $50 \mathrm{ps}$ intervals). The BPM signal shape due to a single bunch is sinusoidal-like, and the TOA is interpolated as the time of the first zero crossing of this signal. The shot-toshot variation in this TOA measurement is due to both intrinsic jitter of the time of flight of the bunches and error on the instrument measurement and interpolation between time samples. We have not quantified each contribution to the measurement variation in TOA but the total typical rms variation is approximately Gaussian with an rms of several ps. By averaging the TOA measurement over many shots a TOA resolution of $<1 \mathrm{ps}$ is obtained, leading to an error of a small fraction of a millimeter on the path length measurement which we expect to be near-normally distributed between measurements.

For each BPM the relative path length can thus be measured as a function of momentum by varying the linac gradient. Since we expect the TOA/path length error to be near-normally distributed between measurements, we would expect this only to add statistical rather than systematic error to the measured path length vs momentum dependence. The linac gradient control parameter is converted into beam momentum using momentum measurements performed with the first dipole of the first arc (which has an independent calibration between current in the coils and field strength) and a downstream screen to measure the beam deflection.

\section{RESULTS}

Using the measurement method described above, the relative path length as a function of beam momentum was measured in the compression section of the lattice, yielding values for $R_{56}$ and $T_{566}$. As described in Sec. III, the outer quadrupoles in the arc provide tunability of the post-linac longitudinal transport and thus, in the first study, the $R_{56}$ and $T_{566}$ were obtained as functions of the quadrupole strengths.

The measurements of path length vs momentum at the arc exit and the chicane exit are shown in Fig. 2. Measurements were made for different arc quadrupole 

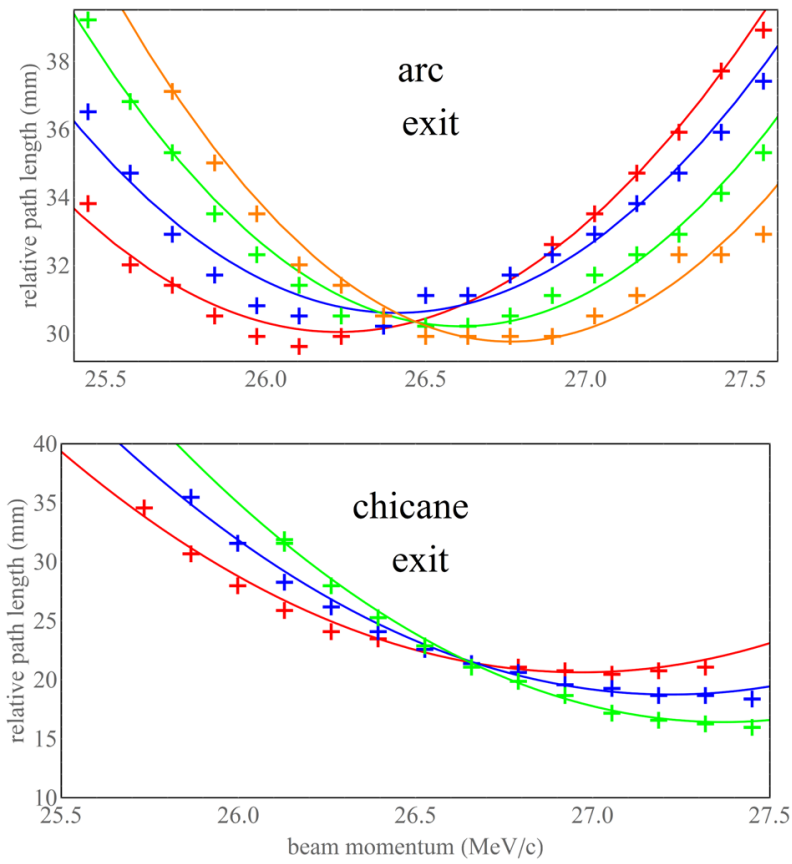

FIG. 2. Relative path length vs beam momentum from linac exit (location B in Fig. 1) to arc exit (location C in Fig. 1) and chicane exit (location D in Fig. 1). Points are measurements, lines are parabolic fits. The colors represent different quadrupole strengths in the first arc, the values of which are indicated in Fig. 3. The nominal ALICE beam momentum is $26.5 \mathrm{MeV} / c$.

strengths (indicated by different colors in Fig. 2). The sextupoles were set to zero strength. The nonlinear nature of the longitudinal transport is very clear. From the curves the values of $R_{56}$ and $T_{566}$ are obtained, as described in Sec. IV, by fitting parabolic curves and calculating the local slope and curvature about the reference momentum $P_{0}=26.5 \mathrm{MeV} / c$. The shapes of the curves at the chicane exit indicate the large $R_{56}$ contribution of the compression chicane; the arc isochronicity condition $\left(R_{56}=\frac{\partial z}{\partial \delta}=0\right)$ is achieved when the bunch momentum is at the minimum of a given parabola in Fig. 2 .

Figure 2 illustrates that different reference momenta (with a fixed lattice) yield different values for $R_{56}$ and $T_{566}$. In other words, if the beam momentum changes then the longitudinal transport will change. Beam momentum "drift" may be possible over a prolonged period of running when the beam momentum is adjusted as an optimization parameter.

For the nominal reference momentum of $26.5 \mathrm{MeV} / c$, the values of $R_{56}$ for the different quadrupole settings are shown in Fig. 3. The errors displayed are the statistical errors obtained from the parabolic fit, which we expect to be dominated be statistical error on the TOA measurement. Shown in the same plot are the theoretical predictions, calculated using an ELEGANT model of the ALICE lattice. The results illustrate the flexibility in the lattice $R_{56}$ provided by the arc quadrupoles, and show that the

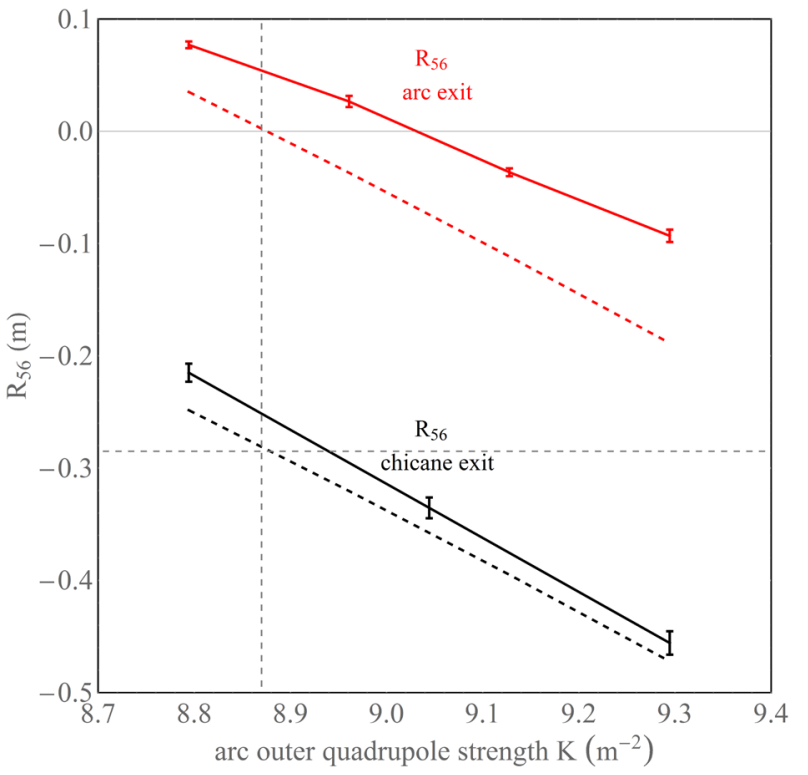

FIG. 3. $R_{56}$ from linac exit to arc exit (red) and from linac exit to chicane exit (black) vs arc quadrupole strength. The dashed lines show the theoretical predictions and the points show the measurements. The reference momentum is $26.5 \mathrm{MeV} / c$. The error bars are the errors from the parabolic fits used to extract the $R_{56}$. The faint dashed vertical and horizontal lines indicate the theoretical closed arc condition where the arc is both isochronous and achromatic.

compression chicane provides an $R_{56}$ contribution $\approx 30 \mathrm{~cm}$, independent of quadrupole strength (as expected and described in Sec. III). At the chicane exit, the change of $R_{56}$ when computed from the measurement with different reference momenta is approximately $12 \mathrm{~cm}$ for a $1 \%$ change in reference momentum (for any of the arc quadrupole strengths in these measurements).

The difference between theory and measurement appears systematic rather than statistical and may come from a number of sources. Since our data does not provide a means to separately determine the individual possible errors we speculate below on some of the likely sources. Errors in magnet field strength affect both theoretical predictions and measurements in multiple ways. Many magnets in ALICE are powered by unipolar power supplies, and cannot be properly degaussed; others may have been degaussed imperfectly. Errors in the magnet field strengths from poor degaussing, or indeed from imperfect magnet strength calibrations, may cause systematic differences between measurement and theory which would be different at the arc exit and the chicane exit. An error in the dipole strength used to measure the beam momentum may also affect the agreement between theory and measurement in a systematic way. Since the measurements were taken on different days, between which the machine had been used for other purposes, the machine setup may not have been identical for both sets of measurements. Using a parabolic fit to the 


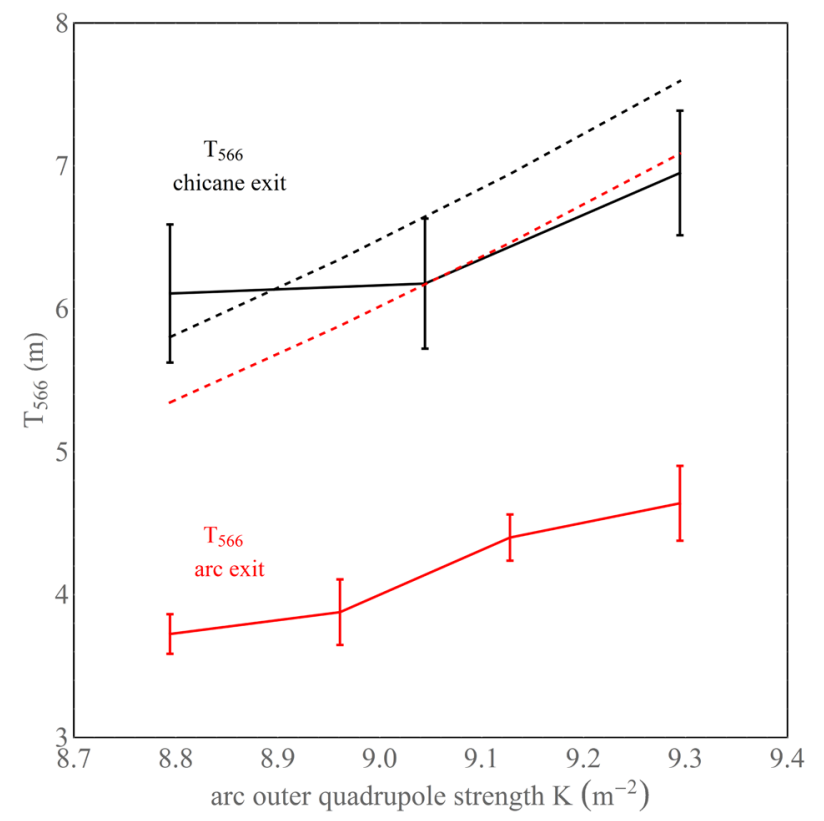

FIG. 4. $T_{566}$ from linac exit to arc exit (red) and from linac exit to chicane exit (black) vs arc quadrupole strength. The dashed lines show the theoretical predictions.

data rather than a higher-order polynomial means that momentum compaction terms higher than second order are neglected; such terms may have a different impact on the longitudinal dynamics between different points in the beam line. Therefore, using a parabolic fit to the data may introduce systematic errors that are different for measurements of the transfer matrix elements to the exit of the arc and to the chicane exit.

The $T_{566}$ of the post-linac lattice (with sextupoles at zero strength) was extracted using the same procedure, and is shown in Fig. 4. There is qualitative-and, partially, quantitative-agreement between the theoretical and the experimentally measured values of $T_{566}$. The causes of systematic error mentioned above similarly affect these results. The "natural" $T_{566}$ (i.e. occurring only from dipoles and quadrupoles) of the lattice would, if uncorrected, increase the distortion of the particle distribution in longitudinal phase space, and could therefore prove detrimental to achieving small bunch length. As described in the preceding sections, sextupoles are located in the arcs to provide control of $T_{566}$, aiding bunch compression and energy recovery. However, sextupoles only provide a pure $T_{566}$ correction when perfectly aligned to the beam; any beam offset will result in the sextupoles also contributing to $R_{56}$.

The effect of the sextupoles on the dependence of path length on momentum clearly demonstrates their impact on the longitudinal dynamics. This is illustrated by Fig. 5, which shows the effect of the first sextupole of the first arc when it is set to its maximum strength (around $50 \mathrm{~T} / \mathrm{m}^{2}$ or, at a reference momentum of $26.5 \mathrm{MeV} / c, \mathrm{~K} \approx 550 \mathrm{~m}^{-3}$ ).

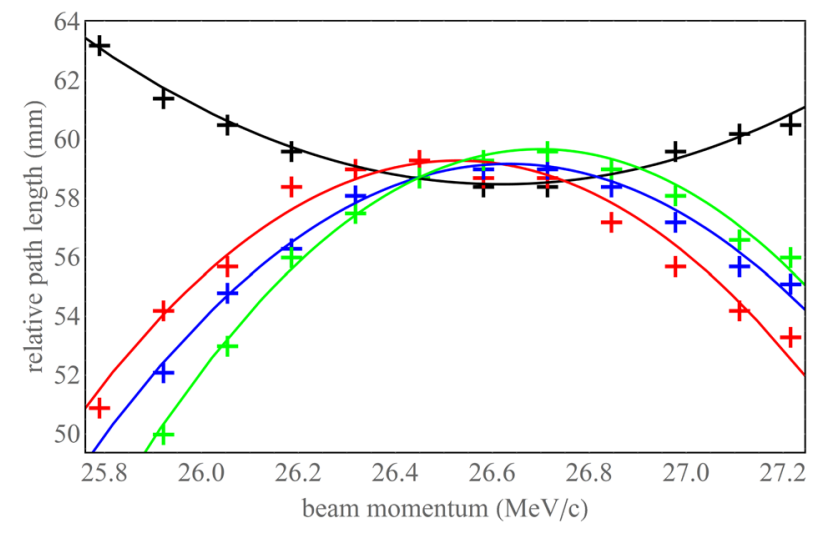

FIG. 5. Relative path length from linac exit to arc exit vs beam momentum, with the first sextupole at zero strength (black line) or at maximum field strength (colored lines). The different colors indicate data taken with different strengths of the first arc dipole, i.e. different beam steering through the first sextupole.

It is clear from the shapes of the curves that the sextupole acts in the opposite sense to the natural $T_{566}$ of the arc, and that unless the beam is set to the appropriate momentum and steered carefully to the center of the sextupole, the $R_{56}$ may be altered. This may not necessarily be advantageous for the final compressed bunch length.

The quantitative effect of the sextupole on the post-linac $R_{56}$ at the reference momentum can be measured as already described, and is shown in Fig. 6. It is clear that the sextupole is capable of changing the post-linac $R_{56}$ by many centimeters in certain circumstances, if the beam is not centered in the sextupole. The dipole strength needed to center the beam in the sextupole (in which case there is no

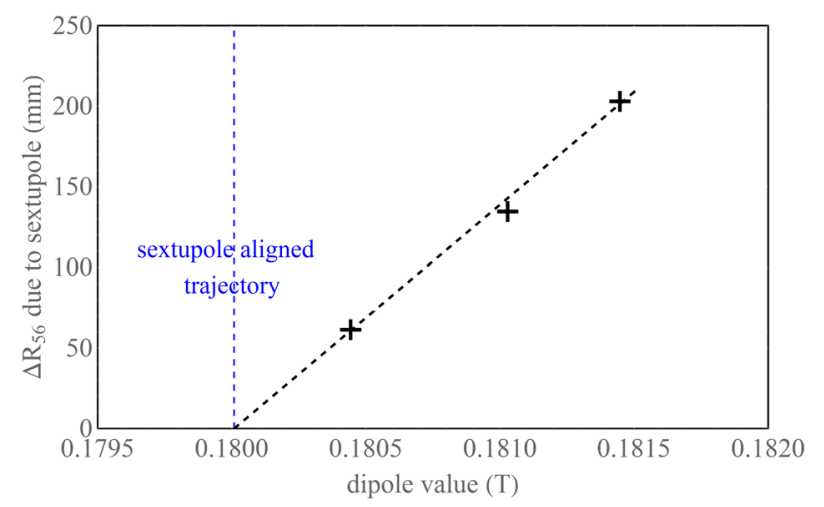

FIG. 6. Change in $R_{56}$ from linac exit to arc exit (at the reference momentum of $26.5 \mathrm{MeV} / c$ ) resulting from increasing the strength of the first arc sextupole from zero to full strength, as a function of the first arc dipole field strength. The points are the data at the three dipole values used in the measurement, the black dashed line is the line of best fit. The dipole strength for the trajectory that passes through the center of the sextupole (in which case there is no change in $R_{56}$ in response to changes in sextupole strength) is indicated by the blue dashed line. 
change in $R_{56}$ in response to changes in sextupole strength) can be found from interpolation of the data. If the arc dipoles are not degaussed carefully and routinely, then a beam-sextupole misalignment of several millimeters may be possible. The measurement may be repeated with the second dipole and second sextupole in the first arc to ensure correct alignment and longitudinal transport properties of the entire arc.

\section{CONCLUSIONS}

The longitudinal transfer matrix elements in the bunch compression section of the ALICE ERL-FEL have been measured and compared against the theoretical design, for various settings of the TBA arc (quadrupole and sextupole strengths). The behavior of $R_{56}$ and $T_{566}$ as functions of the arc quadrupole strengths is close to that expected, confirming the flexibility of the first-order longitudinal transport properties of the TBA arc (and the fixed properties of the chicane). The effects of the sextupoles on $T_{566}$ and (for beams passing off center in the sextupoles) $R_{56}$ have been measured, and confirm the importance of proper beam alignment for achieving well-understood control of the longitudinal dynamics. This emphasizes the need for appropriate diagnostics to enable the necessary alignment to be achieved.

The measurements could be automated to provide rapid online reconstruction of the longitudinal transfer map. This would be useful in operational optimization of the FEL performance, reducing the time needed to tune the FEL and improving the stability over extended running periods. Accurate knowledge of the values of $R_{56}$ and $T_{566}$ would enable fine adjustment (by means of the arc quadrupole and sextupole strengths) to the longitudinal transfer matrix elements on an occasional basis; alternatively, by implementing the results of such measurements in an appropriate feedback system, more frequent corrections could be made. The effectiveness of any correction would depend on appropriate control of the transverse beam alignment in the sextupoles.

\section{ACKNOWLEDGMENTS}

We wish to thank members of ASTeC (Accelerator Science and Technology Centre) and technical departments of STFC (Science and Technology Facilities Council) for providing technical and operational support of the ALICE accelerator, which made these experiments possible.

[1] N. Thompson, D. Dunning, J. Clarke, M. Surman, A. Smith, Y. Saveliev, and S. Leonard, First lasing of the ALICE infra-red free-electron laser, Nucl. Instrum. Methods Phys. Res., Sect. A 680, 117 (2012).
[2] R. Williams, A. Schofield, G. Holder, J. Downes, D. Edgar, P. Harrison, M. Siggel-King, M. Surman, D. Dunning, S. Hill et al., The influence of high intensity terahertz radiation on mammalian cell adhesion, proliferation and differentiation, Phys. Med. Biol. 58, 373 (2013).

[3] P. Piot, D. R. Douglas, and G. A. Krafft, Longitudinal phase space manipulation in energy recovering linacdriven free-electron lasers, Phys. Rev. ST Accel. Beams 6, 030702 (2003).

[4] F. Hug et al., Measurements of a reduced energy spread of a recirculating linac by non-isochronous beam dynamics, in Proceedings of the 26th International Linear Accelerator Conference 2012, Tel-Aviv, Israel (JACoW, Geneva, 2012), TUPB026.

[5] R. Hajima, T. Shizuma, M. Sawamura, R. Nagai, N. Nishimori, N. Kikuzawa, and E. Minehara, First demonstration of energy-recovery operation in the JAERI superconducting linac for a high-power free-electron laser, Nucl. Instrum. Methods Phys. Res., Sect. A 507, 115 (2003).

[6] Y. Shiraga et al., Beam optics study for the compact ERL in Japan, in Proceedings of the 23rd Particle Accelerator Conference, Vancouver, Canada, 2009 (IEEE, Piscataway, NJ, 2009), TU5RFP084.

[7] M. Shimada et al., Bunch compression at the recirculation loop of the compact ERL, in Proceedings of the 7th international Particle Accelerator Conference 2016, Busan, Korea (JACoW, Geneva, 2016), WEPOY010.

[8] E. Antokhin et al., First lasing at the high-power free electron laser at Siberian center for photochemistry research, Nucl. Instrum. Methods Phys. Res., Sect. A 528, 15 (2004).

[9] M. Abo-Bakr et al., Magnet optics and beam dynamics of BERLinPro, in Proceedings of the International Particle Accelerator Conference, Kyoto, Japan (ICR, Kyoto, 2010), TUPD102.

[10] M. Abo-Bakr et al., Progress report of the Berlin energy recovery project BERLinPro, in Proceedings of the 6th International Particle Accelerator Conference 2015, Richmond, Virginia, USA (JACoW, Geneva, 2015), TUPWA018.

[11] H. Ohgaki, I. Tometaka, K. Yamane, T. Kii, K. Masuda, K. Yoshikawa, and T. Yamazaki, Design studies of IR-FEL system at IAE, Kyoto University, Nucl. Instrum. Methods Phys. Res., Sect. A 507, 150 (2003).

[12] S. Benson et al., First lasing of the Jefferson Lab IR Demo FEL, Nucl. Instrum. Methods Phys. Res., Sect. A 429, 27 (1999).

[13] B. Muratori et al., Optics layout for the ERL Prototype at Daresbury Laboratory, in Proceedings of the 9th European Particle Accelerator Conference, Lucerne, 2004 (EPS-AG, Lucerne, 2004), MOPKF061.

[14] H. L. Owen et al., Choice of Arc Design for the ERL Prototype at Daresbury Laboratory, in Proceedings of the 9th European Particle Accelerator Conference, Lucerne, 2004 (Ref. [13]), MOPKF062.

[15] C. Gerth et al., Start-to-end simulations of the energy recovery linac prototype FEL, in Proceedings of FEL2004 Conference (Comitato Conferenze Elettra, Trieste, Italy, 2004), THPOS47. 
[16] E. T. d'Amico and G. Guignard, Special lattice computation for the CERN Compact Linear Collider, Phys. Rev. ST Accel. Beams 4, 021002 (2001).

[17] H. Grote and F. Schmidt, MAD-X: An upgrade from MAD8, in Proceedings of the 2003 Particle Accelerator Conference, Portland, OR (IEEE, New York, 2003), FPAG014.
[18] M. Borland, ELEGANT: A flexible SDDS-compliant code for accelerator simulation, Advanced Photon Source Report No. LS-287, 2000.

[19] H. L. Owen and P. H. Williams, A modular path length corrector for recirculating linacs, Nucl. Instrum. Methods Phys. Res., Sect. A 662, 12 (2012). 